\title{
OPEN Preparation of self-healing hydrogel toward improving electromagnetic interference shielding and energy efficiency
}

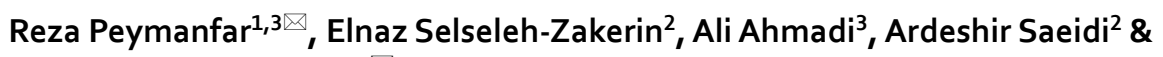 \\ Seyed Hassan Tavassoli ${ }^{1 \bowtie}$
}

In this study, a self-healing hydrogel was prepared that is transparent to visible (Vis) light while absorbing ultraviolet (UV), infrared (IR), and microwave. The optothermal features of the hydrogel were explored by monitoring temperature using an IR thermometer under an IR source. The hydrogel was synthesized using sodium tetraborate decahydrate (borax) and polyvinyl alcohol (PVA) as raw materials based on a facile thermal route. More significantly, graphene oxide (GO) and graphite-like carbon nitride $\left(\mathrm{g}-\mathrm{C}_{3} \mathrm{~N}_{4}\right.$ ) nanostructures as well as carbon microsphere (CMS) were applied as guests to more dissect their influence on the microwave and optical characteristics. The morphology of the fillers was evaluated using field emission scanning electron microscopy (FE-SEM). Fourier transform infrared (FTIR) attested that the chemical functional groups of the hydrogel have been formed and the result of diffuse reflection spectroscopy (DRS) confirmed that the hydrogel absorbs UV while is transparent in Vis light. The achieved result implied that the hydrogel acts as an essential IR absorber due to its functional groups desirable for energy efficiency and harvesting. Interestingly, the achieved results have testified that the self-healing hydrogels had the proper self-healing efficiency and selfhealing time. Eventually, microwave absorbing properties and shielding efficiency of the hydrogel, hydrogel/GO, g- $\mathrm{C}_{3} \mathrm{~N}_{4}$, or CMS were investigated, demonstrating the salient microwave characteristics, originated from the established ionic conductive networks and dipole polarizations. The efficient bandwidth of the hydrogel was as wide as $3.5 \mathrm{GHz}$ with a thickness of $0.65 \mathrm{~mm}$ meanwhile its maximum reflection loss was $75.10 \mathrm{~dB}$ at $14.50 \mathrm{GHz}$ with $4.55 \mathrm{~mm}$ in thickness. Particularly, the hydrogel illustrated total shielding efficiency $\left(\mathrm{SE}_{\mathrm{T}}\right)>10 \mathrm{~dB}$ from 1.19 to 18 and $>20 \mathrm{~dB}$ from 4.37 to $18 \mathrm{GHz}$ with $10.00 \mathrm{~mm}$ in thickness. The results open new windows toward improving the shielding and energy efficiency using practical ways.

Recently, artificial intelligence has been the hotspot, leading to the revolution in the strategy of architecting the technologies in the present era. Smart devices are an inseparable part of the body of artificial intelligence. The smart devices are constructed from the high-tech subsets, needing technologies using the high frequencies to transport data ${ }^{1}$. Thus, electronic devices applying high frequencies have been developed toward facilitating our inescapable mechanical life. The charge circuits in electronic boards can establish the secondary fields and electromagnetic leakages which can be hazardous for any living species. On the other hand, the wireless devices employing electromagnetic waves have augmented our interactions by electromagnetic pollution. Thereby, to avoid receiving electromagnetic pollution by humans, microwave absorbing and shielding materials have been tailored. The permittivity and permeability of the absorbing or shielding structures play vital roles, paving the way for the microwave attenuation, as given by the transmission line theor $\mathrm{y}^{2-12}$. Therefore, diverse structures have been applied to provide these features. Among them, the carbonaceous structures have been extensively used due to their significant polarization and conductive loss features. The achieved results manifest that the morphology of the aforementioned structures plays a vital role in regulating their microwave absorbing and shielding characteristics. The carbon nanotube, fiber, wire, sphere, graphene, flake, graphene foam, carbide, net,

${ }^{1}$ Laser and Plasma Research Institute, Shahid Beheshti University, G. C., Evin, 19839 Tehran, Iran. ${ }^{2}$ Department of Polymer Engineering, Science and Research Branch, Islamic Azad University, Tehran, Iran. ${ }^{3}$ Department of Chemical Engineering, Energy Institute of Higher Education, Saveh, Iran. ${ }^{凶}$ email: reza_peymanfar@ alumni.iust.ac.ir; h-tavassoli@sbu.ac.ir 
as well as other carbonaceous structures derived from the pyrolysis of the metal-organic framework (MOF)s and biomass-derived materials have attracted a great deal of attention in recent years ${ }^{13-22}$. On the other hand, the absorbing matrix is the crucial parameter tuning the microwave characteristics. Paraffin wax as a conventional polymeric medium was used to investigate the microwave characteristics of the fabricated structures while its composites suffer from the proper mechanical properties. Other matrices including polystyrene, polyvinylidene fluoride, polymethylmethacrylate, silicone rubber, and so on have been widely used to strengthen mechanical properties of the shielding or absorbing structures and reinforce their microwave features ${ }^{19,20,23-25}$. These media promote the practical applications of the microwave absorbing and shielding composites in environmental, industrial, and military fields. Recent works have illustrated that the promising scenarios have been developed providing the novel perspective of microwave absorbers. Cao et al. have fabricated liquid crystalline elastomer and investigated its microwave characteristics ${ }^{26}$. Ionic conductive gels, which were transparent in visible light at room temperature, were architected by Fang et al. using phosphoric acid and PVA, demonstrating the significant microwave absorbing characteristics ${ }^{27}$. Recently, borax-crosslinked PVA hydrogel system with three-dimensional (3D) hierarchical network structures has demonstrated fascinating tissue-like, moldable, viscoelastic, biodegradable, flexible, tunable solvent content, biocompatible, mechanical, and lightweight properties. Noticeably, the characteristics of the aforementioned self-healing hydrogel were promoted by diverse fillers comprising polyaniline, carbon nanotube, polypyrrole, nanocellulose, etc. toward improving its intelligent applications in new-generation supercapacitors with smart functions, wearable and portable electronics, flexible and even self-reparative energy storage/conversion devices, soft bioelectronics and biosensors, electro-stimulated drug release devices and electronic skin, as well as bioelectrodes and strain sensors with excellent electrochemical performances ${ }^{28-33}$. Among them, cellulose-based structures were extensively applied in modern technologies including flexible/transparent substrates, batteries, super capacitors, electroactive materials in flexible sensors, and triboelectric nanogenerators due to their outstanding biocompatibility, low-cost, processability, biodegradability, and good mechanical flexibility. Particularly, this phenomenon is mainly originated from the potential of this type of material to modulate the chemical, structural, dielectric, and optical properties ${ }^{34}$. Thus, diverse approaches have been employed to modify the surface, defect, interface, polarizability, and chemical structure of nanocellulosic precursors for advanced materials ${ }^{35}$.

As known, the global warming has been the main challenge, exciting the global concern about the climate change. Improving the energy efficiency is the dominant way to prevent the global warming. In order to overcome the issue, the building materials have been developed based on their climate ${ }^{36}$.

Interestingly, various methods have been applied to reinforce the building materials against the electromagnetic waves ${ }^{36}$. It should be noted that the conventional electromagnetic shielding (ES) materials benefit from the impedance mismatching elevating the reflection at their interface. Thus, ES structures were architected based on the absorption, diminishing secondary hazards. Noteworthy, not only the large number of hydroxyl groups existing in the hydrogel are desirable for energy and ES efficiency, by absorbing IR waves and dipole polarization, but also by establishing hydrogen bonds with hydroxyl and amine groups related to CMS, GO, and g- $\mathrm{C}_{3} \mathrm{~N}_{4}$ act as dispersing agents generating uniform composites. More significantly, the malleability of the hydrogel composites on one hand develop their practical applications as interlayer filler of laminated glass or double glazed windows on the other hand the self-healing feature of the 3D scaffolds reinforce their optical characteristics. Particularly, the ionic conductivity of the tailored hydrogels plays the key role, amplifying their microwave characteristics. In this study, a novel approach has been presented that not only diminishes energy consumption in the tropical regions but also enhances the ES efficiency based on absorption. A transparent self-healing hydrogel was fabricated through a facile route using borax as a raw material which can be used in our windows elevating energy and shielding efficiency.

\section{Experimental}

Materials. D-glucose (97.5-102.0\%), sulfuric acid (95.0-97.0\%), hydrochloric acid fuming (37.0\%), and hydrogen peroxide $(30.0 \%)$ were purchased from Merck meanwhile urea $(98.0 \%)$, potassium permanganate (99.3\%), sodium nitrate (99.0\%), and borax 99.0-103.0\%) were supplied from Samchun Chemicals. Moreover, PVA and graphite nanoparticles were obtained from Japan Vam \& Poval Co. (POVAL ${ }^{\mathrm{ma}}$ JP-24) and US Research Nanomaterials, Inc. (C, $400 \mathrm{~nm}-1.2 \mu \mathrm{m}, 99.9 \%)$, respectively.

Experimental steps. Preparation of the fillers. The $\mathrm{g}-\mathrm{C}_{3} \mathrm{~N}_{4}, \mathrm{GO}$, and CMS as the fillers were prepared using the reported literature ${ }^{23,37-39}$.

Fabrication of self-healing structures. PVA $5 \mathrm{wt} \%$ was dissolved in deionized water and the $\mathrm{g}-\mathrm{C}_{3} \mathrm{~N}_{4}$, GO, and CMS (guest/borax + PVA =2 wt\%) were separately suspended in the solution, following that the aforementioned suspension was simultaneously sonicated and stirred by an over head stirrer for $30 \mathrm{~min}$. Afterwards, the borax (borax $/ \mathrm{PVA}=20 \mathrm{wt} \%$ ) was dissolved in deionized water and added to the solution. The self-healing composites as novel microwave absorbers were synthesized by keeping the samples at $90^{\circ} \mathrm{C}$ for $3 \mathrm{~h}$. A self-healing hydrogel was prepared without any filler to compare the results, based on the presented procedures. Figure 1 depicts a schematic illustration of the synthetic procedure applied to prepare self-healing hydrogels.

Characterization. The microwave characteristics were estimated by Agilent technology instrument (E8364A). The optothermal characteristics were examined by an IR source and monitored by an IR thermometer Lutron TM-958. The chemical species were identified using Shimadzu 8400 as well as Shimadzu MPC-2200 evaluated optical performance of the hydrogel at $\lambda=200-800 \mathrm{~nm}$. MIRA3TESCAN-XMU revealed the morphology of guests. Stress-strain behavior of the samples was investigated by MCR 502 rheometer from Anton Paar. 


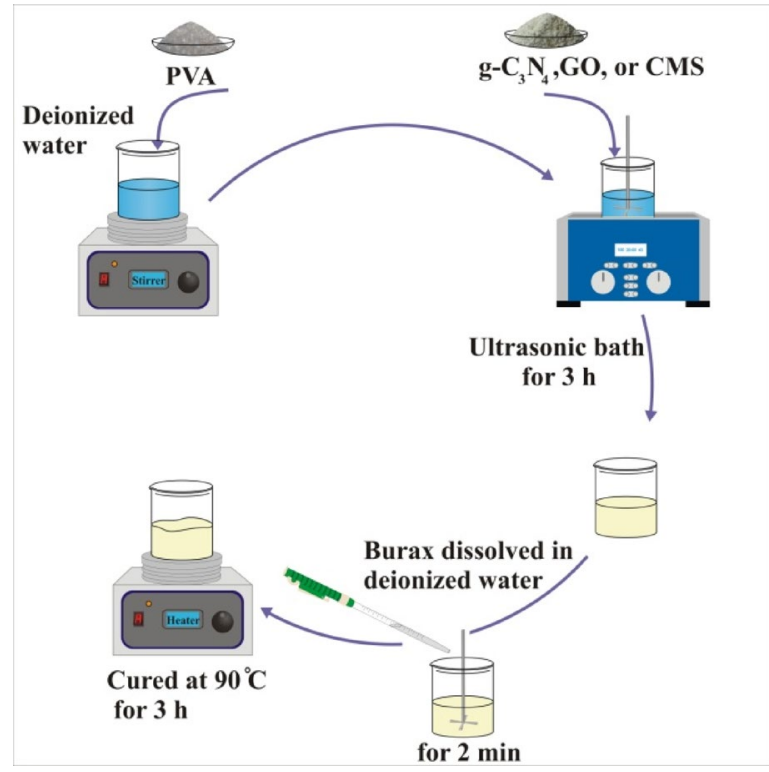

Figure 1. A schematic illustration of the synthetic procedure applied to prepare self-healing hydrogels.
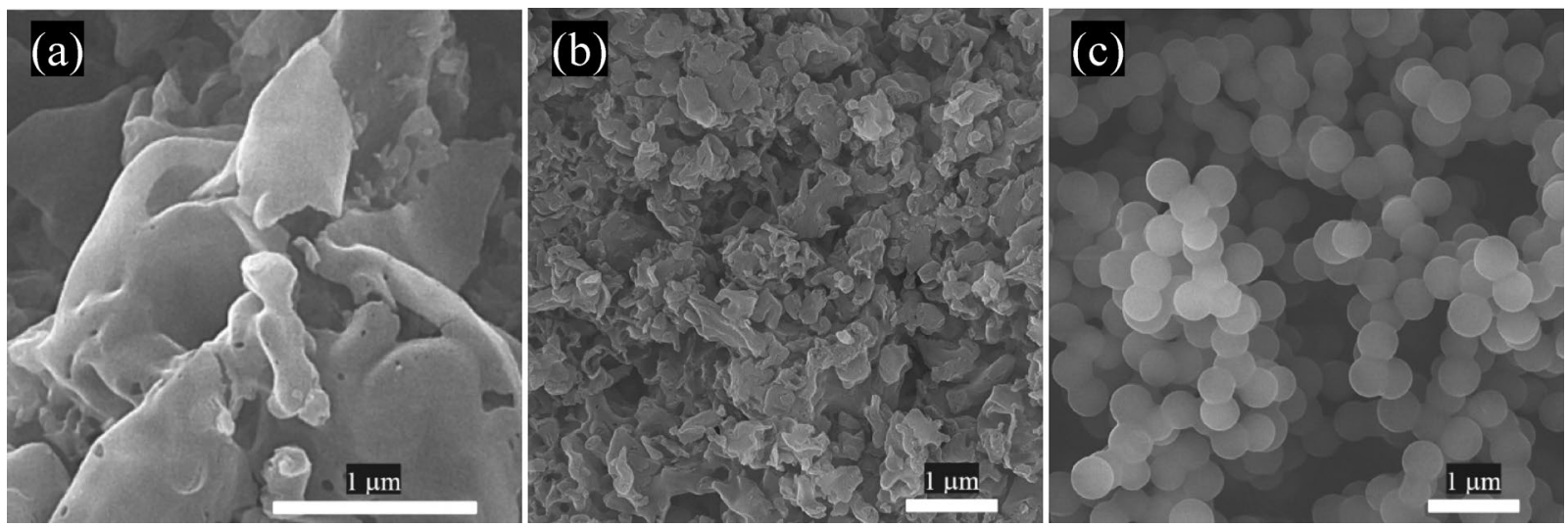

Figure 2. FESEM micrographs of $\mathrm{GO}(\mathbf{a}), \mathrm{g}-\mathrm{C}_{3} \mathrm{~N}_{4}(\mathbf{b})$, and $\mathrm{CMS}(\mathbf{c})$.

\section{Results and discussions}

Morphology. Figure 2 illustrates FESEM micrographs of the guests comprising GO, g- $\mathrm{C}_{3} \mathrm{~N}_{4}$, and CMS. As indicated, all of the fillers have a uniform and smooth morphology. It can be seen that the self-assembly scenario of D-glucose along the hydrothermal process led to the formation of CMS with an average diameter of $600 \mathrm{~nm}^{20}$. More interestingly, 2D nanostructures with proper exfoliation and smooth surface of GO and g- $\mathrm{C}_{3} \mathrm{~N}_{4}$ have been synthesized. It is well known that the more surface area to volume ratio augments the interfacial interactions in heterogeneous structures, desirable for relaxation loss mechanism ${ }^{24,39}$.

Chemical species. Figure 3 presents FTIR spectrum of the samples. The assigned peak at 3400 and $1636 \mathrm{~cm}^{-1}$ are respectively ascribed to the stretching and bending vibrations of the hydroxyl functional groups existing in the hydrogel meanwhile the peaks at 2920 and 2850, and $802 \mathrm{~cm}^{-1}$ are related to the symmetric and asymmetric stretching vibrations as well as out-of-plane bending vibrations of $\mathrm{C}-\mathrm{H}$ functional groups ${ }^{14,18,31,33,40,41}$. Besides, the observed peaks at $1419,1273,1109$, and bump at $716 \mathrm{~cm}^{-1}$ are associated with the stretching vibrations of $\mathrm{B}-\mathrm{O}$ and $\mathrm{C}-\mathrm{O}$ derivates including $\mathrm{B}-\mathrm{O}-\mathrm{C}, \mathrm{B}-\mathrm{O}-\mathrm{H}, \mathrm{C}-\mathrm{O}-\mathrm{H}$, and $\mathrm{C}-\mathrm{O}-\mathrm{B}$, as well as deformation vibration of $\mathrm{O}-\mathrm{B}-\mathrm{O}$ in the borate networks ${ }^{42-46}$. It can be seen that some of the peaks related to the conjugated structures have overlapped by the characteristics peaks of hydrogel ${ }^{14,20,39}$.

Optical performance. The optical performance of the hydrogel, hydrogel/GO, hydrogel/g- $\mathrm{C}_{3} \mathrm{~N}_{4}$, and hydrogel/CMS were investigated from $\lambda=200$ to $800 \mathrm{~nm}$ displayed in Figs. 4 and S1. The light absorption is mainly originated by the charge transmissions from the valance band to the conduction band. Evidently, the fabricated hydrogel is almost transparent in the Vis light meanwhile it can significantly absorb the UV light, confirmed by its energy band gap ${ }^{25}$. Particularly, the sample absorbs more than $80 \%$ of the UV light, implying 


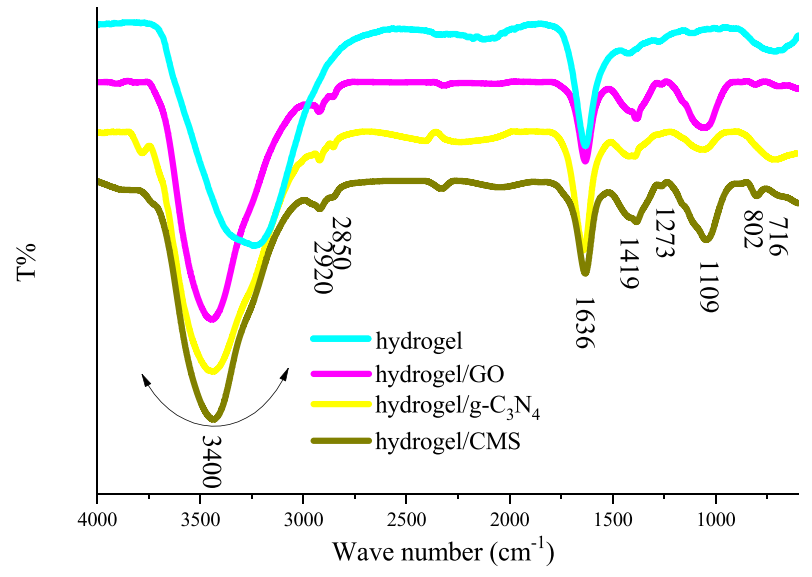

Figure 3. FTIR spectra of the samples.
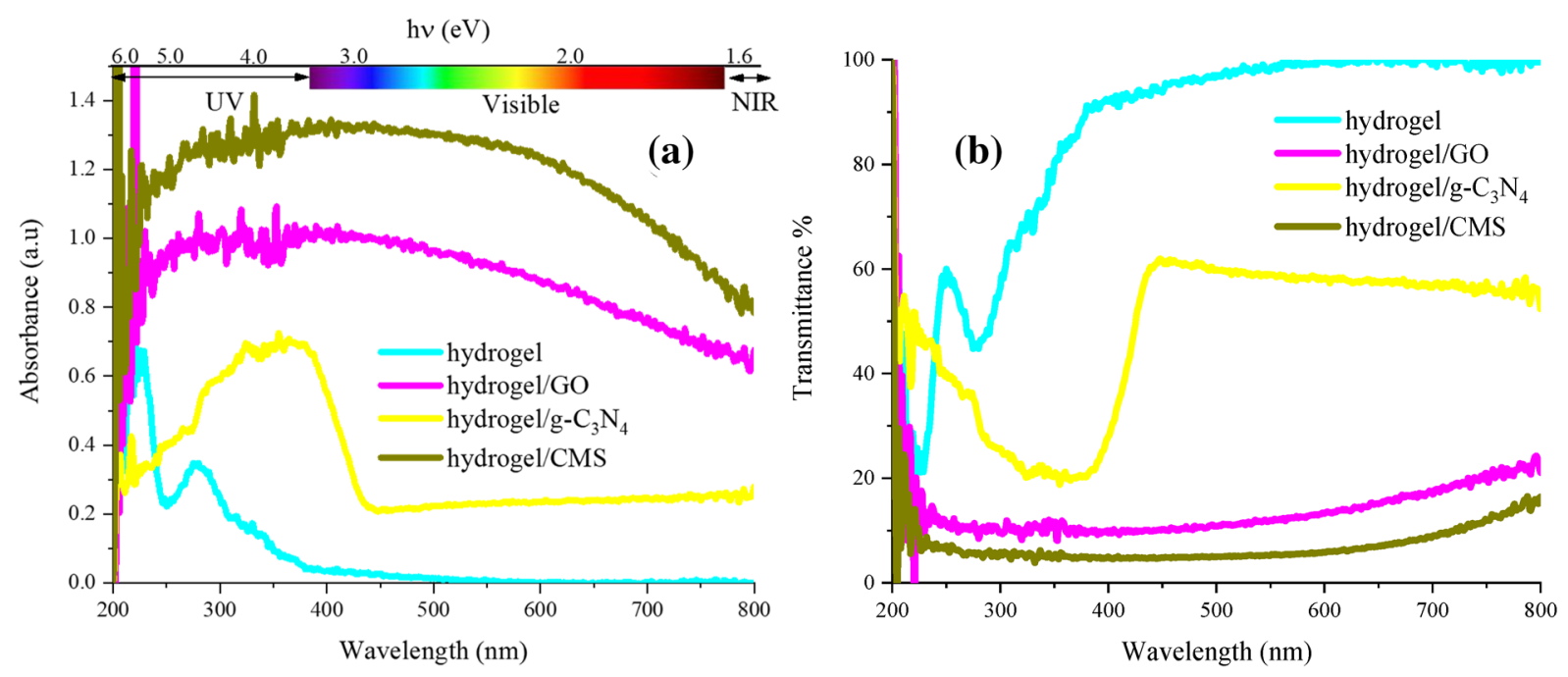

Figure 4. The light absorption (a) and transmittance $\%$ at $\lambda=200-800 \mathrm{~nm}$ (b) of the samples.

the importance of the presented research in the practical application against UV light. The $\mathrm{n} \rightarrow \pi^{\star}$ transitions could be a dominant parameter to produce the observed UV light absorption of the self-healing hydrogel. The displayed photographs refer to the visible transparency of the pure hydrogel meanwhile the loaded structures diminished the transparency of the fabricated structures (Fig. 4). The energy band gaps were evaluated based on Kubelka-Munk theory, given by the following equation: $(\alpha h v)^{2}=h v-E g, \alpha=-1 / t \ln T$, and $T=10^{-\mathrm{A}}$, where $v, A, t, h, T, \alpha$, and $E_{g}$ refer to the frequency, absorbance, thickness, Planck constant, transmittance, absorption coefficient, and energy gap. The achieved results clarified that the energy band gaps of hydrogel, hydrogel/ $\mathrm{GO}$, hydrogel/g- $\mathrm{C}_{3} \mathrm{~N}_{4}$, and hydrogel/CMS were $3.90,1.59,2.92$, and $1.44 \mathrm{eV}$, respectively. It is found that the inserted structures have regulated the distance between the highest occupied molecular orbital (HOMO) and lowest unoccupied molecular orbital (LUMO) of the fabricated structures, mainly generated from their intrinsic characteristics $^{20,39}$.

Self-healing property and mechanical performance. Figure 5 depicts the self-healing procedure and stress-strain curve of the self-healing hydrogels. The fabricated self-healing hydrogels were cut into two equal parts. The separated blocks were contacted together at fractured zone without external stress or additional stimuli for $12 \mathrm{~s}$ at room temperature. Noticeably, the self-healing hydrogels were wholly merged. It is noteworthy that the self-healing time of the tailored self-healing hydrogels is so lower than that of polyacrylic acid-based self-healing structures ${ }^{40,41,47,48}$. This spontaneous phenomenon is originated from diffusion and permeation at fractured interfaces, generated from the molecular dynamics reviving inter- and intramolecular hydrogen bonds, healing cross-links, and leading to more and more entanglements of the polymers ${ }^{28,29,31-33}$. Hydroxyl functional groups are mainly associated with PVA, CMS, GO, and water meanwhile amine groups come from $\mathrm{g}_{-} \mathrm{C}_{3} \mathrm{~N}_{4}$, producing hydrogen bonds. More importantly, the established tetrahedron $\mathrm{B}(\mathrm{OH})_{4}{ }_{4}^{-}$acts as cross-linking agent. Tensile strength $(F)$ and self-healing efficiency $\left(\eta_{F}\right.$ values of the self-healing hydrogels were summarized 


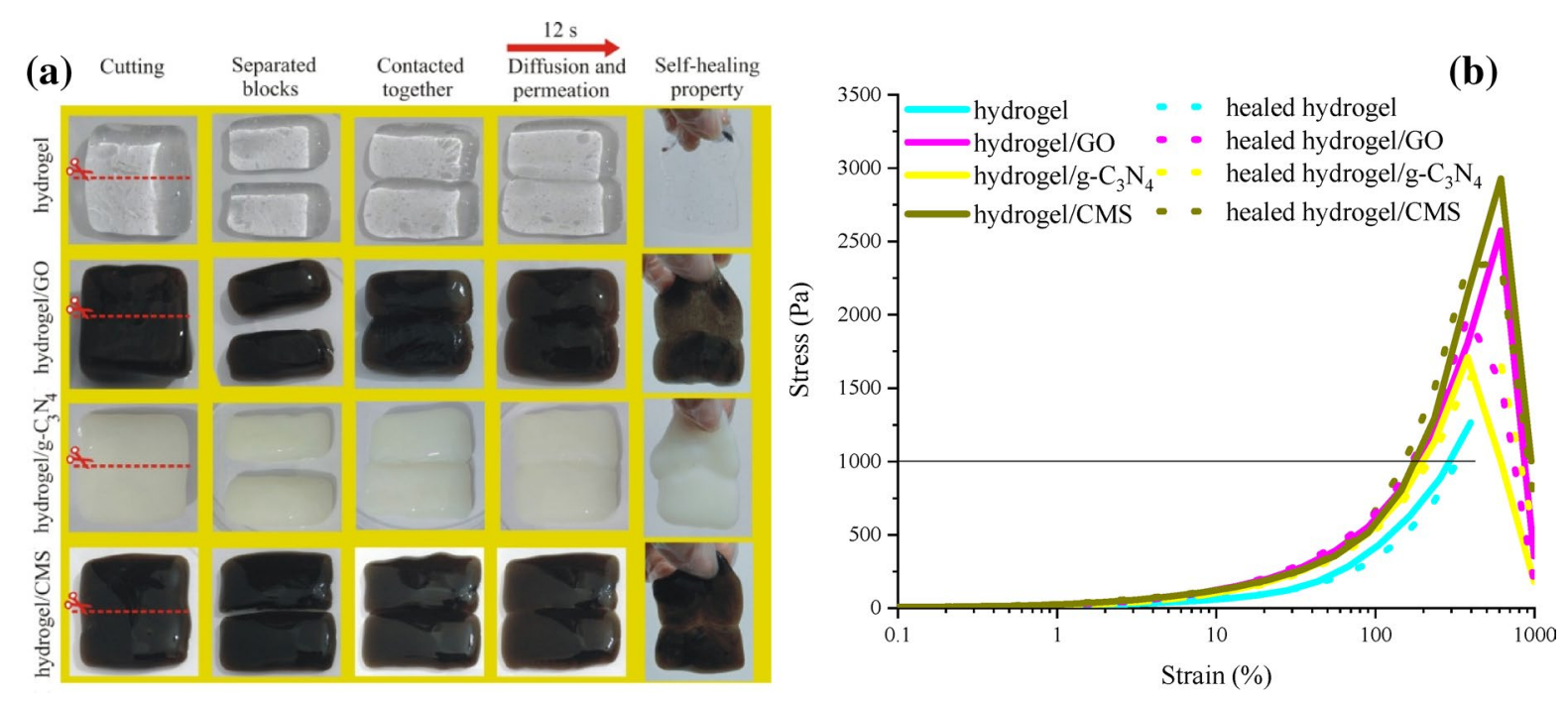

Figure 5. Self-healing procedure (a) and stress-strain curve (b) of the hydrogels.

\begin{tabular}{|l|l|l|l|}
\hline Sample & $\mathbf{F}_{\text {original }}(\mathbf{P a})$ & $\mathbf{F}_{\text {healed }}(\mathbf{P a})$ & $\boldsymbol{\eta}_{\mathbf{F}}(\%)$ \\
\hline Hydrogel & 1259.5 & 1114.7 & 88.5 \\
\hline Hydrogel/GO & 2567.5 & 1981.1 & 77.2 \\
\hline Hydrogel/g- $\mathrm{C}_{3} \mathrm{~N}_{4}$ & 1703.8 & 1662.0 & 97.5 \\
\hline Hydrogel/CMS & 2922.7 & 2342.4 & 80.1 \\
\hline
\end{tabular}

Table 1. F and self-healing parameter of the samples.

in Table 1 where $\eta_{\mathrm{F}}$ is evaluated by the following equation $\eta_{\mathrm{F}} \%=\left(\mathrm{F}_{\text {healed }} / \mathrm{F}_{\text {original }}\right) 100^{32,33}$. As revealed, at a stress of $1000 \mathrm{~Pa}$ the elongation of samples was ordered as hydrogel $>$ hydrogel/g- $\mathrm{C}_{3} \mathrm{~N}_{4}>$ hydrogel/GO $>$ hydrogel/CMS meanwhile the tensile strengths were hydrogel/CMS $>$ hydrogel/GO $>$ hydrogel/g- $\mathrm{C}_{3} \mathrm{~N}_{4}>$ hydrogel, derived from the emerged chemical interactions into the self-healing media.

Optothermal features. The abundant portion of the sunlight is IR (about 50\%), warming the earth along a day but it is improper to the tropical region. This phenomenon not only augments energy consumption due to keeping cool our buildings but also the used energy enhances the greenhouse effect creating secondary damages. Figure 6 exhibits IR light absorption of the fabricated hydrogel and the architected setup used to investigate the optothermal features. As indicated, a setup was architected to investigate the practical application of the hydrogel. Briefly, a cube was constructed using commercial glass with $0.4 \mathrm{~cm}$ thickness and $10.0 \times 10.0 \times 1.8 \mathrm{~cm}$ dimensions and then it was filled by the hydrogel. Afterwards, the fabricated cube was placed between the IR source and an aluminium plate $(10.0 \times 10.0 \times 0.1 \mathrm{~cm}$ dimensions $)$. Next, the temperature of the filled cube and aluminium plate under the IR source was monitored by an IR thermometer. Noteworthy, warming the glass and aluminium plate without the shielder were evaluated to more dissect and compare the results. It can be seen that the filled cube is warmed faster and more than the bare glass cube. On the other hand, the achieved results attested that the aluminium plate behind the filled glass cube was colder than the aluminium plate without shielder or with bare glass cube shielder. The obtained results manifest that the chemical structure of transparent self-healing hydrogel acts as an IR absorber, confining penetration of the incident waves. Therefore, the designed hydrogel can be applied as a novel structure in tropical regions keeping cool the buildings, desirable for improving energy efficiency.

Microwave absorbing and shielding features. Figures 7 and S2 presents the real part of permittivity $\left(\varepsilon^{\prime}\right)$, imaginary part of permittivity $\left(\varepsilon^{\prime \prime}\right)$, microwave absorption, maximum reflection loss versus matching thickness, efficient bandwidth $(\mathrm{RL}>10 \mathrm{~dB})$ versus matching thickness, and simulation of matching thickness for the samples from 1 to $18 \mathrm{GHz}$. The real part was originated from the storage of the waves meanwhile the imaginary part came from the dissipation of them. $\mathrm{GO}, \mathrm{g}-\mathrm{C}_{3} \mathrm{~N}_{4}$, and CMS were employed as guests to more examine their influence on the microwave features of the hydrogel. Evidently, by enhancing the frequency the real parts of the samples are descended nevertheless their imaginary parts are ascended. The permittivity of the hydrogel is mainly generated from its dipole polarization, electron hopping, and established ionic conductive network. Microwave absorptions of the samples were evaluated by the transmission line theory ${ }^{49-51}$. As given by the results, the efficient bandwidth of the hydrogel was as wide as $3.5 \mathrm{GHz}$ with only a thickness of $0.65 \mathrm{~mm}$ meanwhile its maximum reflection loss was $75.10 \mathrm{~dB}$ at $14.50 \mathrm{GHz}$ with $4.55 \mathrm{~mm}$ in thickness. It can be seen that 

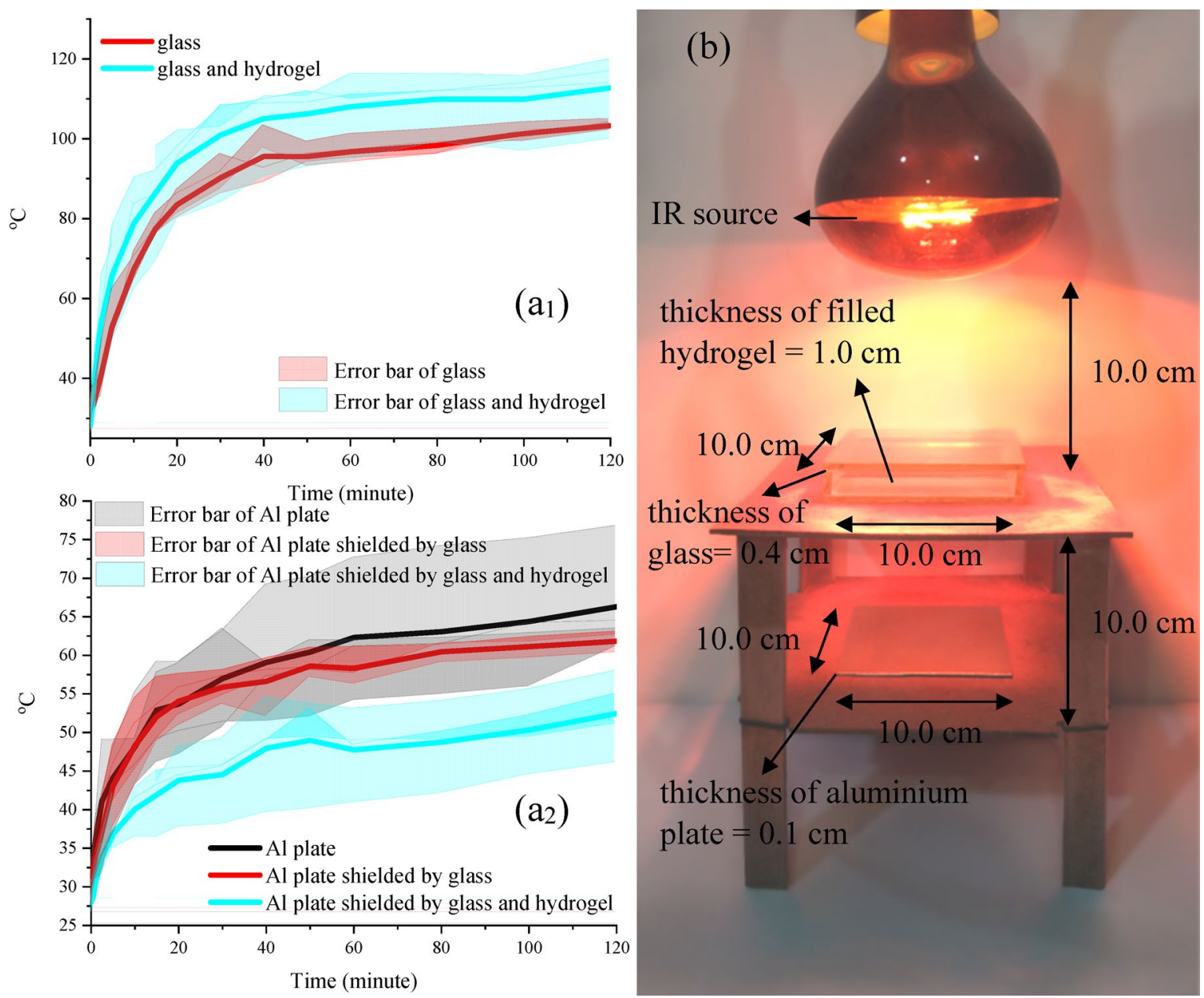

Figure 6. IR light absorption $\left(\mathbf{a}_{1,2}\right)$ and optothermal setup (b).

the quarter wavelength mechanism is the dominant parameter bringing microwave absorption of the samples, defined by the attenuated waves by the reversal waves when they are $180^{\circ}$ out of phase of incident waves and the thickness of the absorber is an odd numeral of the $\lambda / 4$ of penetrated waves ${ }^{52}$. Noteworthy, the type of fillers modulates the maximum reflection loss and efficient bandwidth, derived from their unique characteristics including conductive loss as well as interfacial and dipole polarization. However, the obtained results manifest that the hydrogel performs the essential duty for the microwave absorbing features. Impedance matching $(\mathrm{Z})$ is the crucial factor that has a clear trade-off between the propagation of the incident waves in the absorbing medium $(Z=1)$. As revealed, not all samples have the proper impedance matching, but loading the fillers augmented $Z$. The attenuation constant $(\alpha)$ implies the potential of an absorber for energy conversion that has a clear compromise with imaginary parts of the permittivity and permeability ${ }^{53}$. Figure 8 displays $\mathrm{Z}$ and $\alpha$ of the microwave absorbing structures.

$\mathrm{SE}_{\mathrm{T}}$ is obtained from the sum of the shielding efficiency of absorbance $\left(\mathrm{SE}_{\mathrm{A}}\right)$ and reflectance $\left(\mathrm{SE}_{\mathrm{R}}\right)^{54}$. Obviously, $\mathrm{SE}_{\mathrm{R}}$ is independent of the thickness as confirmed by its equation. All of the samples exhibited considerable $\mathrm{SE}_{\mathrm{T}}$, established by their $\mathrm{SE}_{\mathrm{A}}$. Interestingly, absorbance plays a vital role in paving the way for the shielding property of the samples containing hydrogel. This phenomenon diminishes the secondary pollution, produced by the reflectance at the interface of the shielding structures. Particularly, the hydrogel illustrated total shielding efficiency $\mathrm{SE}_{\mathrm{T}}>10 \mathrm{~dB}$ from 1.19 to 18 and $>20 \mathrm{~dB}$ from 4.37 to $18 \mathrm{GHz}$ with $10.00 \mathrm{~mm}$ in thickness. Figure 9 exposes $\mathrm{SE}_{\mathrm{R}}, \mathrm{SE}_{\mathrm{A}}$, and $\mathrm{SE}_{\mathrm{T}}$ of the samples.

Based on the Debye relaxation theory, each emerged semicircle, produced by drawing $\varepsilon^{\prime}$ versus $\varepsilon^{\prime \prime}$ refers to one relaxation process, known as Cole-Cole $\operatorname{plot}^{55}$. The plots attest that all of the samples follow the distinct pattern confirming that only one type of polarization exists in the absorbing media, generated from the dipole polarization and charge transfer in the self-healing hydrogel. Skin depth $(\delta)$ is the dominant factor regulating SE of structures ${ }^{56-58}$. This factor is modulated by alternative conductivity $\left(\sigma_{\mathrm{AC}}\right)$ which is tuned by $\varepsilon^{\prime \prime 59}$. Thus, the imaginary part of the permittivity plays a crucial role in modulating SE of the shielding materials. There are $\mathrm{n} \rightarrow \sigma^{*}$ transitions from the lone pair electrons of oxygen and $\sigma^{*}$ of hydrogen coupled to the oxygen (hydrogen bonds). As indicated, $\sigma_{\mathrm{AC}}$ is enhanced by elevating the frequency implying that by increasing the energy the charge transitions, originated from the ionic conductivity and relaxation loss are promoted. Figure 10 depicts the Cole-Cole plot, $\delta$, and $\sigma_{\mathrm{AC}}$ for the tailored specimens. Figure 11 exhibits an illustrative diagram of the commanding mechanisms in the hydrogel. The above-mentioned equations were presented in the supporting information. 

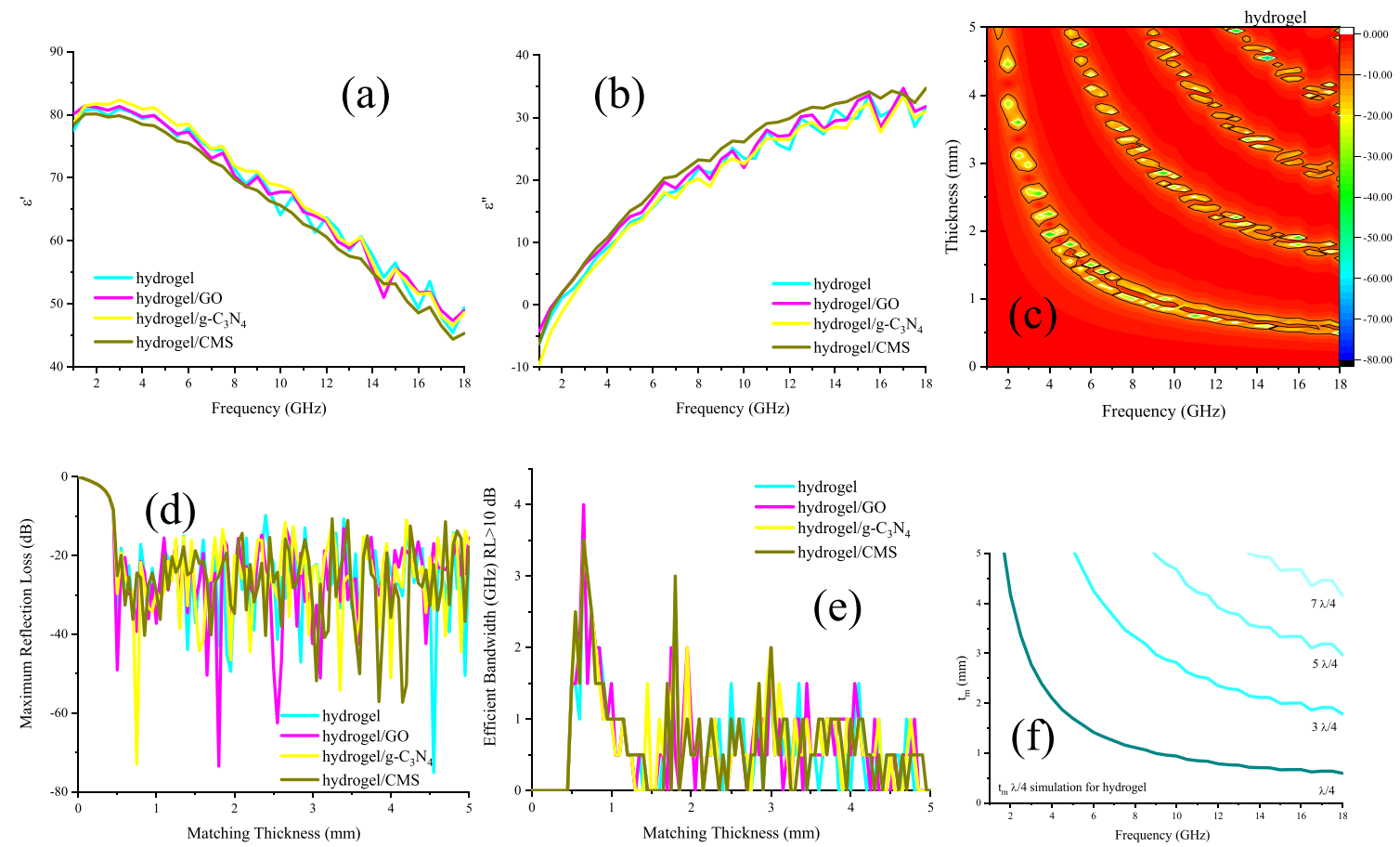

Figure 7. $\varepsilon^{\prime}(\mathbf{a}), \varepsilon^{\prime \prime}(\mathbf{b})$, microwave absorption (c), maximum reflection loss versus matching thickness (d), efficient bandwidth $(\mathrm{RL}>10 \mathrm{~dB})$ versus matching thickness $(\mathbf{e})$, and simulation of matching thickness $(\mathbf{f})$ for the samples from 1 to $18 \mathrm{GHz}$.
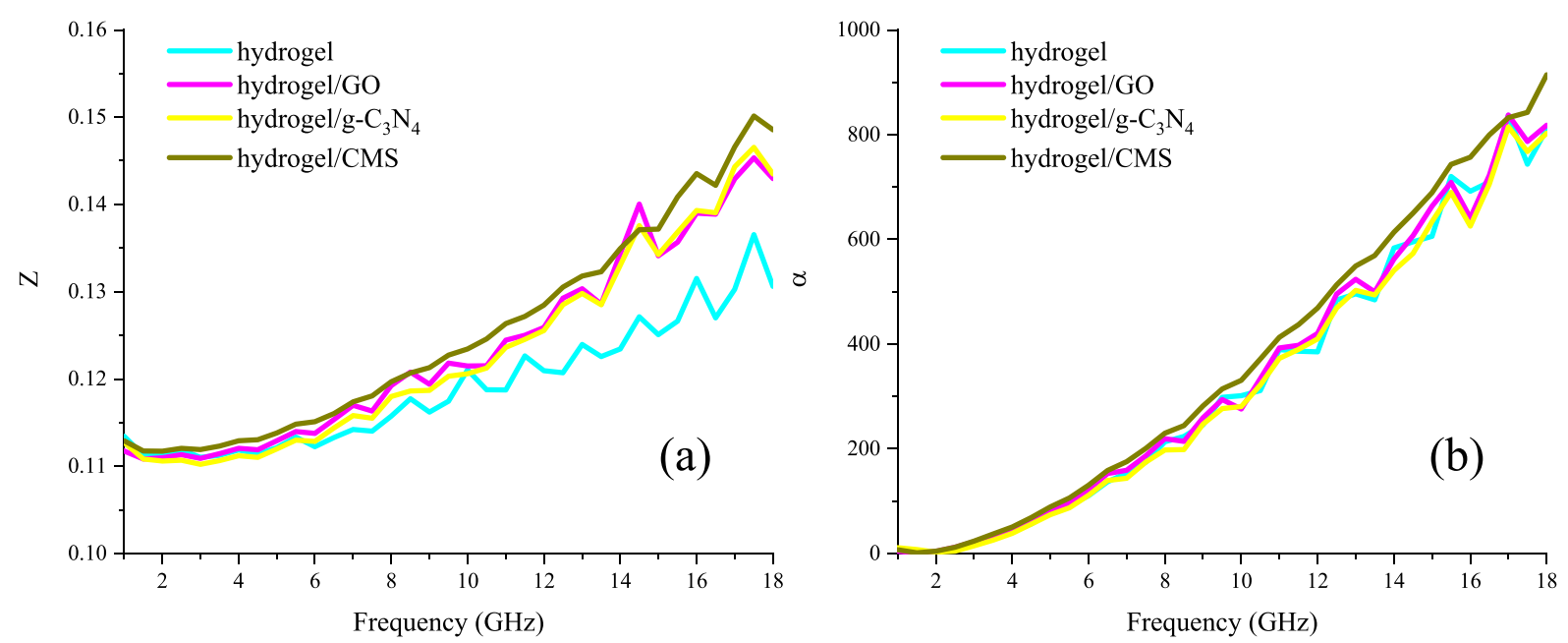

Figure 8. $\mathrm{Z}(\mathbf{a})$ and $\alpha(\mathbf{b})$ of the microwave absorbing structures.

\section{Conclusion}

In this research, a self-healing hydrogel was fabricated which was transparent in Vis light while absorbing IR, UV, and microwave. This ability opens new windows toward reinforcing our building against harmful microwave irradiations by using the hydrogel in windows. Interestingly, the hydrogel illustrated considerable IR absorption attesting to its ability to apply in the tropics, keeping our buildings cool through its use in windows, for example. Diverse fillers were applied to more dissect the microwave absorbing and shielding characteristics. It should be noted that the obtained shielding results of the samples were based on the absorption diminishing the secondary pollution established by the reflection at the shielding interface. It can be seen that the nanofillers and CMSs have no significant influence on microwave characteristics. The achieved results manifest that the dipole polarization, electron hopping, and produced ionic conductive network are the pioneer mechanisms bringing microwave properties of the structures. 

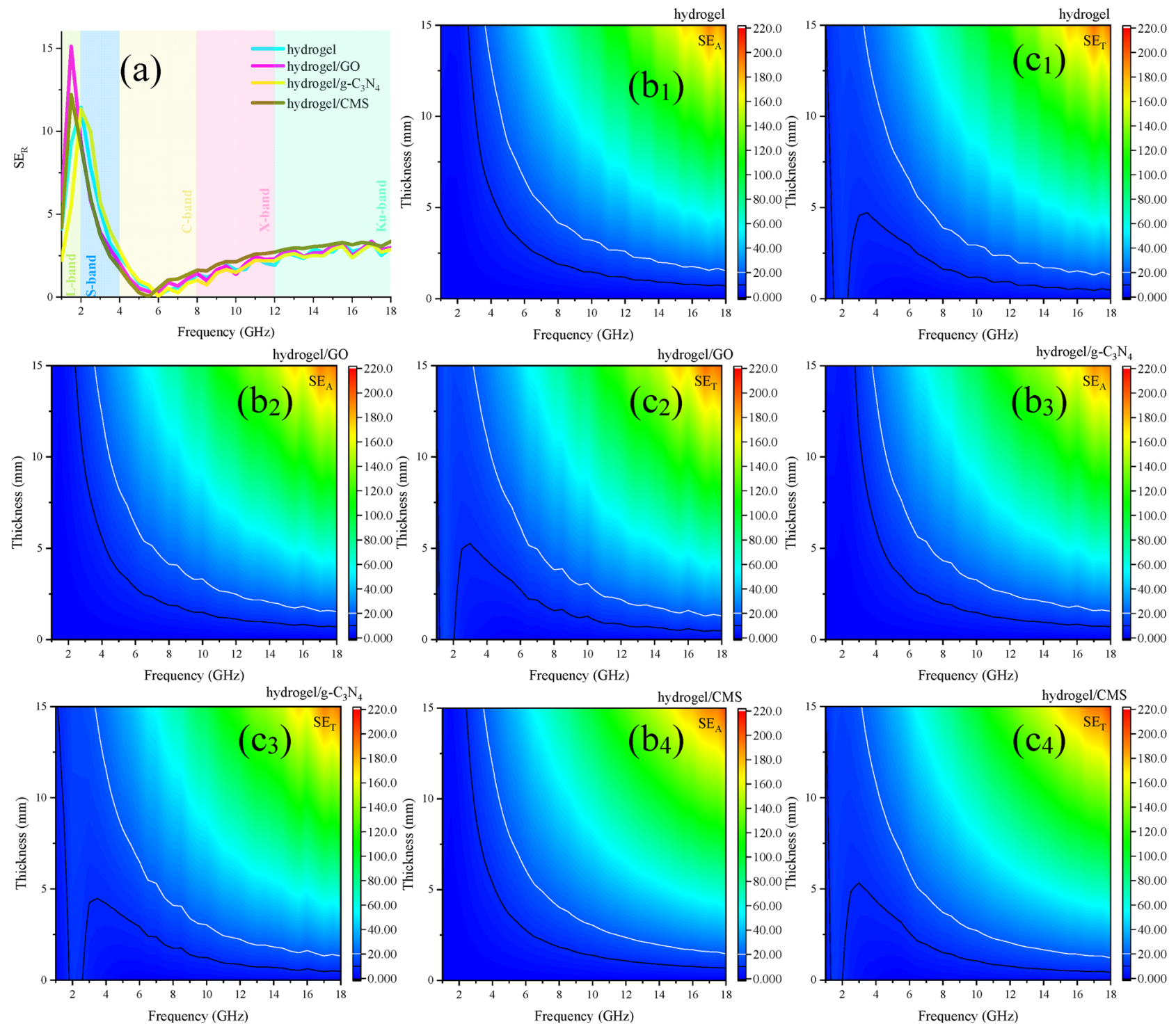

Figure 9. $\mathrm{SE}_{\mathrm{R}}(\mathbf{a}), \mathrm{SE}_{\mathrm{A}}\left(\mathbf{b}_{1-4}\right)$, and $\mathrm{SE}_{\mathrm{T}}\left(\mathbf{c}_{1-4}\right)$ of the hydrogel containing structures. 

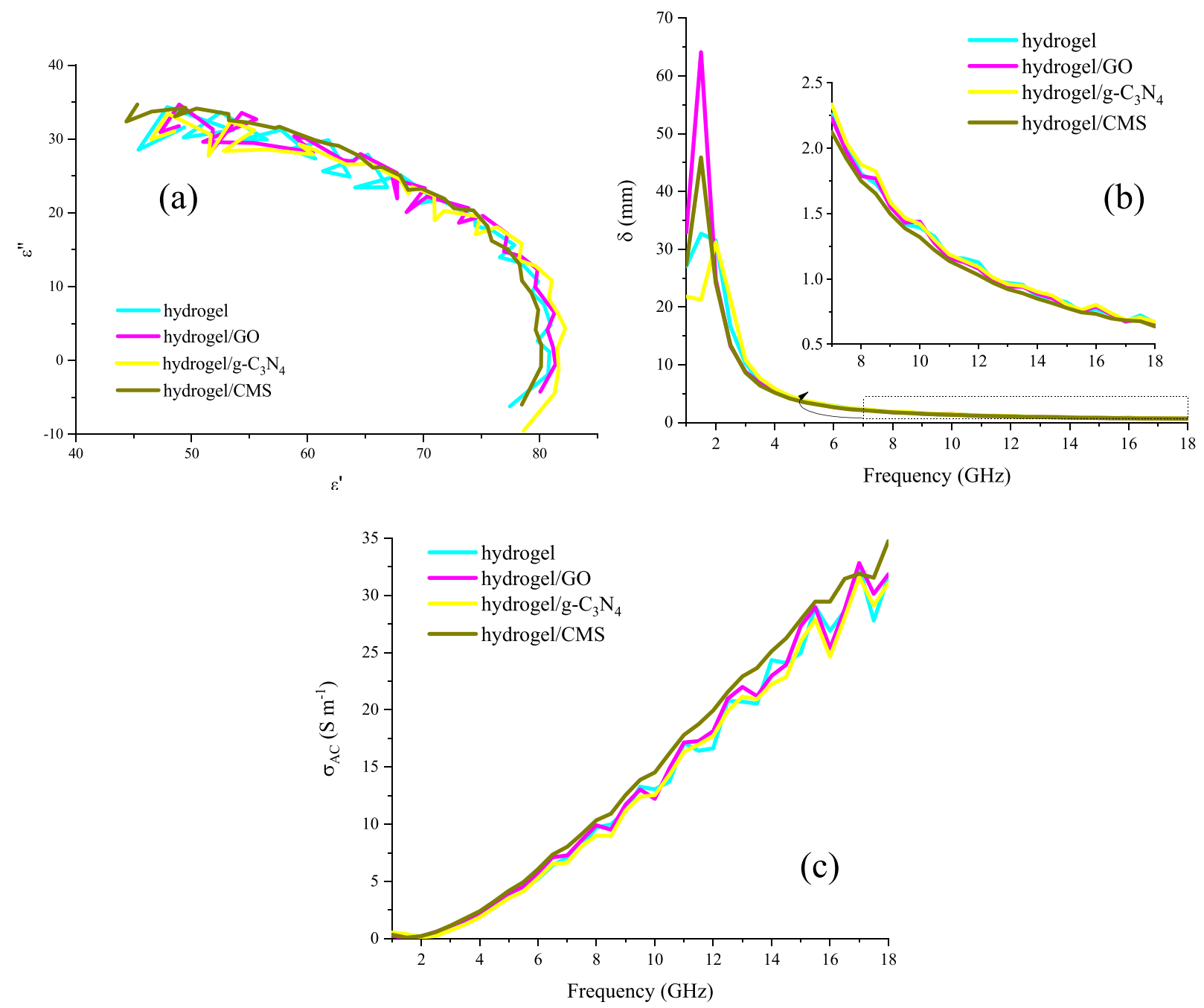

Figure 10. Cole-Cole plot (a), $\delta(\mathbf{b})$, and $\sigma_{\mathrm{AC}}(\mathbf{c})$ for the prepared samples. 


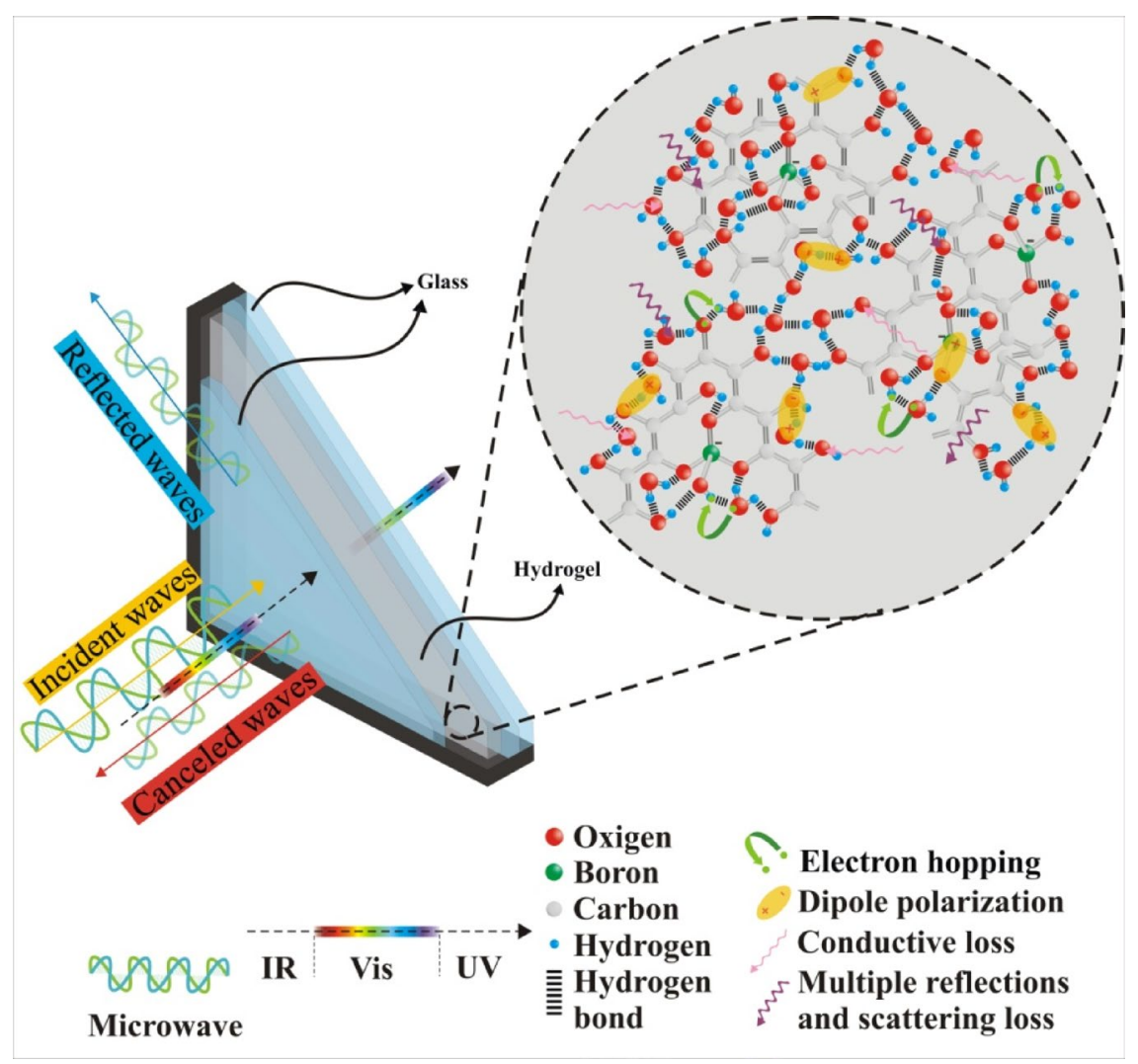

Figure 11. An illustrative diagram of the commanding mechanisms in the hydrogel.

Received: 22 May 2021; Accepted: 29 July 2021

Published online: 09 August 2021

\section{References}

1. Wang, X. X., Cao, W. Q., Cao, M. S. \& Yuan, J. Assembling nano-microarchitecture for electromagnetic absorbers and smart devices. Adv. Mater. 1, $2002112(2020)$.

2. Cao, M. S. et al. Electronic structure and electromagnetic properties for $2 \mathrm{D}$ electromagnetic functional materials in gigahertz frequency. Ann. Phys. 1, 1800390 (2019).

3. Peymanfar, R. \& Rahmanisaghieh, M. Preparation of neat and capped $\mathrm{BaFe} 2 \mathrm{O} 4$ nanoparticles and investigation of morphology, magnetic, and polarization effects on its microwave and optical performance. Mater. Res. Express 5, 105012 (2018).

4. Peymanfar, R. \& Azadi, F. Preparation and identification of bare and capped $\mathrm{CuFe} 2 \mathrm{O} 4$ nanoparticles using organic template and investigation of the size, magnetism, and polarization on their microwave characteristics. Nano-Struct. Nano-Objects 17, 112-122 (2019).

5. Peymanfar, R., Javanshir, S., Naimi-Jamal, M. R. \& Cheldavi, A. Preparation and identification of modified La0.8Sr0.2FeO3 nanoparticles and study of its microwave properties using silicone rubber or PVC. Mater. Res. Express 6, 075004 (2019).

6. Cao, M. S. et al. Electromagnetic response and energy conversion for functions and devices in low-dimensional materials. $A d v$. Funct. Mater. 29, 1807398 (2019).

7. Wu, Z. et al. Enhanced microwave absorption performance from magnetic coupling of magnetic nanoparticles suspended within hierarchically tubular composite. Adv. Funct. Mater. 29, 1901448 (2019).

8. He, P. et al. atomic layer tailoring titanium carbide MXene to tune transport and polarization for utilization of electromagnetic energy beyond solar and chemical energy. ACS Appl. Mater. Interfaces 11, 12535-12543 (2019).

9. Chen, H. et al. Graphene-based materials toward microwave and terahertz absorbing stealth technologies. Adv. Opt. Mater. 7, 1801318 (2019).

10. Zhang, D. et al. Lightweight and high-performance microwave absorber based on 2D WS 2-RGO heterostructures. Nano-Micro Lett. 11, 38 (2019).

11. Li, Q. et al. Toward the application of high frequency electromagnetic wave absorption by carbon nanostructures. Adv. Sci. 6, 1801057 (2019).

12. Zhao, H. et al. Biomass-derived porous carbon-based nanostructures for microwave absorption. Nano-Micro Lett. 11, 24 (2019).

13. Peymanfar, R. \& Ghorbanian-Gezaforodi, S. Functionalized carbonized monarch butterfly wing scales (FCBW) ornamented by $\beta$-Co (OH) 2 nanoparticles: An investigation on its microwave, magnetic, and optical characteristics. Nanotechnology 32, 195201 (2021).

14. Peymanfar, R. \& Fazlalizadeh, F. Fabrication of expanded carbon microspheres/ZnAl2O4 nanocomposite and investigation of its microwave, magnetic, and optical performance. J. Alloys Compd. 854, 157273 (2020).

15. Wang, X. X., Cao, W. Q., Cao, M. S. \& Yuan, J. Assembling nano-microarchitecture for electromagnetic absorbers and smart devices. Adv. Mater. 32, $2002112(2020)$.

16. Han, C., Zhang, M., Cao, W.-Q. \& Cao, M.-S. Electrospinning and in-situ hierarchical thermal treatment to tailor C- $\mathrm{NiCo}_{2} \mathrm{O}_{4}$ nanofibers for tunable microwave absorption. Carbon 171, 953-962 (2021).

17. Balci, O., Polat, E. O., Kakenov, N. \& Kocabas, C. Graphene-enabled electrically switchable radar-absorbing surfaces. Nat. Commun. 6, 1-10 (2015). 
18. Peymanfar, R. \& Moradi, F. Functionalized carbon microfibers (biomass-derived) ornamented by Bi2S3 nanoparticles: An investigation on their microwave, magnetic, and optical characteristics. Nanotechnology 32, 065201 (2020).

19. Peymanfar, R. \& Ghorbanian-Gezaforodi, S. Preparation of graphite-like carbon nitride (g-C3N4)/NiCo2S4 nanocomposite toward salient microwave characteristics and evaluation of medium influence on its microwave features. Nanotechnology 31, 495202 (2020).

20. Peymanfar, R. et al. Electromagnetic and optical characteristics of wrinkled Ni nanostructure coated on carbon microspheres. Chem. Eng. J. 405, 126985 (2020).

21. Wang, G., Ong, S. J. H., Zhao, Y., Xu, Z. J. \& Ji, G. Integrated multifunctional macrostructures for electromagnetic wave absorption and shielding. J. Mater. Chem. A 8, 24368-24387 (2020).

22. Peymanfar, R., Selseleh-Zakerin, E., Ahmadi, A. \& Tavassoli, S. H. Architecting functionalized carbon microtube/carrollite nanocomposite demonstrating significant microwave characteristics. Sci. Rep. 11, 1-15 (2021).

23. Peymanfar, R., Karimi, J. \& Fallahi, R. Novel, promising, and broadband microwave-absorbing nanocomposite based on the graphite-like carbon nitride/CuS. J. Appl. Polym. Sci. 137, 48430 (2019).

24. Peymanfar, R. \& Fazlalizadeh, F. Microwave absorption performance of ZnAl2O4. Chem. Eng. J. 402, 126089 (2020).

25. Peymanfar, R., Yektaei, M., Javanshir, S. \& Selseleh-Zakerin, E. Regulating the energy band-gap, UV-Vis light absorption, electrical conductivity, microwave absorption, and electromagnetic shielding effectiveness by modulating doping agent. Polymer 209, 122981 (2020).

26. Wang, X. et al. A study of the microwave actuation of a liquid crystalline elastomer. Soft Matter 16, 7332-7341 (2020).

27. Song, W. L. et al. Ionic conductive gels for optically manipulatable microwave stealth structures. Adv. Sci. 7, 1902162 (2020).

28. Zheng, C. et al. Highly stretchable and self-healing strain sensors based on nanocellulose-supported graphene dispersed in electroconductive hydrogels. Nanomaterials 9,937 (2019).

29. Ding, Q. et al. Nanocellulose-mediated electroconductive self-healing hydrogels with high strength, plasticity, viscoelasticity, stretchability, and biocompatibility toward multifunctional applications. ACS Appl. Mater. Interfaces. 10, 27987-28002 (2018).

30. Han, J. et al. Effects of nanocellulose on the structure and properties of poly (vinyl alcohol)-borax hybrid foams. Cellulose 24, 4433-4448 (2017).

31. Han, J. et al. An intrinsically self-healing and biocompatible electroconductive hydrogel based on nanostructured nanocellulosepolyaniline complexes embedded in a viscoelastic polymer network towards flexible conductors and electrodes. Electrochim. Acta 318, 660-672 (2019).

32. Wang, H. et al. Self-healable electro-conductive hydrogels based on core-shell structured nanocellulose/carbon nanotubes hybrids for use as flexible supercapacitors. Nanomaterials 10, 112 (2020).

33. Han, J. et al. A self-healable and highly flexible supercapacitor integrated by dynamically cross-linked electro-conductive hydrogels based on nanocellulose-templated carbon nanotubes embedded in a viscoelastic polymer network. Carbon 149, 1-18 (2019).

34. Niu, Z. et al. Recent advances in cellulose-based flexible triboelectric nanogenerators. Nano Energy 87, 106175 (2021).

35. Yang, X. et al. Surface and interface engineering for nanocellulosic advanced materials. Adv. Mater. 33, 2002264 (2020).

36. Peymanfar, R., Keykavous-Amand, S., Abadi, M. M. \& Yassi, Y. A novel approach toward reducing energy consumption and promoting electromagnetic interference shielding efficiency in the buildings using Brick/polyaniline nanocomposite. Constr. Build. Mater. 263, $120042(2020)$.

37. Peymanfar, R., Ahmadi, M. \& Javanshir, S. Tailoring GO/BaFe12O19/La0.5Sr0.5MnO3 ternary nanocomposite and investigation of its microwave characteristics. Mater. Res. Express 6, 085063 (2019).

38. Sun, X. \& Li, Y. Colloidal carbon spheres and their core/shell structures with noble-metal nanoparticles. Angew. Chem. Int. Ed. 43, 597-601 (2004).

39. Peymanfar, R., Selseleh-Zakerin, E. \& Ahmadi, A. Tailoring energy band gap and microwave absorbing features of graphite-like carbon nitride (g-C3N4). J. Alloys Compd. 867, 159039 (2021).

40. Zheng, C. et al. A stretchable, self-healing conductive hydrogels based on nanocellulose supported graphene towards wearable monitoring of human motion. Carbohydr. Polym. 250, 116905 (2020).

41. Jiao, Y. et al. Highly stretchable and self-healing cellulose nanofiber-mediated conductive hydrogel towards strain sensing application. J. Colloid Interface Sci. 597, 171-181 (2021).

42. Huang, S. et al. Facile fabrication and characterization of highly stretchable lignin-based hydroxyethyl cellulose self-healing hydrogel. Carbohydr. Polym. 223, 115080 (2019).

43. Lu, B. et al. One-pot assembly of microfibrillated cellulose reinforced PVA-borax hydrogels with self-healing and $\mathrm{pH}$-responsive properties. ACS Sustain. Chem. Eng. 5, 948-956 (2017).

44. Dixit, A., Bag, D. S. \& Kalra, S. Synthesis of strong and stretchable double network (DN) hydrogels of PVA-borax and P (AM-coHEMA) and study of their swelling kinetics and mechanical properties. Polymer 119, 263-273 (2017).

45. Huang, M., Hou, Y., Li, Y., Wang, D. \& Zhang, L. High performances of dual network PVA hydrogel modified by PVP using borax as the structure-forming accelerator. Des. Monomers Polym. 20, 505-513 (2017).

46. Bian, H. et al. Lignin nanoparticles as nano-spacers for tuning the viscoelasticity of cellulose nanofibril reinforced polyvinyl alcohol-borax hydrogel. Eur. Polymer J. 107, 267-274 (2018).

47. Chen, Y. et al. A skin-inspired stretchable, self-healing and electro-conductive hydrogel with a synergistic triple network for wearable strain sensors applied in human-motion detection. Nanomaterials $\mathbf{9}, 1737$ (2019).

48. Jiao, Y. et al. Highly viscoelastic, stretchable, conductive, and self-healing strain sensors based on cellulose nanofiber-reinforced polyacrylic acid hydrogel. Cellulose 28, 4295-4311 (2021).

49. Shu, R., Li, W., Wu, Y., Zhang, J. \& Zhang, G. Nitrogen-doped Co-C/MWCNTs nanocomposites derived from bimetallic metalorganic frameworks for electromagnetic wave absorption in the X-band. Chem. Eng. J. 362, 513-524 (2019).

50. Qiang, R. et al. Metal organic framework-derived Fe/C nanocubes toward efficient microwave absorption. J. Mater. Chem. A 3, 13426-13434 (2015).

51. Hussein, M. I. et al. Microwave absorbing properties of metal functionalized-CNT-polymer composite for stealth applications. Sci. Rep. 10, 1-11 (2020).

52. Zhu, Y. et al. Large-scale preparation of Co nanoparticles as an additive in carbon fiber for microwave absorption enhancement in C band. Sci. Rep. 11, 1-14 (2021).

53. Ibrahim, I. R. et al. A study on microwave absorption properties of carbon black and $\mathrm{Ni0} .6 \mathrm{Zn} 0.4 \mathrm{Fe} 2 \mathrm{O} 4$ nanocomposites by tuning the matching-absorbing layer structures. Sci. Rep. 10, 1-14 (2020).

54. Liu, X. et al. Facile fabrication of ultrathin graphene film with ultrahigh electrical conductivity and superb electromagnetic interference shielding effectiveness. J. Mater. Chem. C 9, 214-222 (2021).

55. Gu, W. et al. Extending effective microwave absorbing bandwidth of CoNi bimetallic alloy derived from binary hydroxides. Sci. Rep. 10, 1-11 (2020).

56. Zhou, M. et al. Sustainable wood-based composites for microwave absorption and electromagnetic interference shielding. J. Mater. Chem. A 8, 26467 (2020).

57. Qiao, M. et al. Well-defined core-shell Fe3O4@ polypyrrole composite microspheres with tunable shell thickness: Synthesis and their superior microwave absorption performance in the Ku band. Ind. Eng. Chem. Res. 55, 6263-6275 (2016).

58. Mustaffa, M. S., Azis, R. A. S., Abdullah, N. H., Ismail, I. \& Ibrahim, I. R. An investigation of microstructural, magnetic and microwave absorption properties of multi-walled carbon nanotubes/Ni0.5Zn0.5Fe2O4. Sci. Rep. 9, 15523. https://doi.org/10.1038/ s41598-019-52233-2 (2019). 
59. Lv, J. et al. Nanofiber network with adjustable nanostructure controlled by PVP content for an excellent microwave absorption. Sci. Rep. 9, 1-10 (2019).

\section{Acknowledgements}

This work is supported by Iran Science Elites Federation Grant No. 11/66332.

\section{Author contributions}

R.P.: Project administration, Supervision, Methodology, Visualization, Resources, Conceptualization, Data curation, Writing-review \& editing, Drawing Figs. 2, 3, 4, 5, 6, 7, 8, 9, and 10 except 5a. E.S.-Z.: Formal analysis, Investigation, Resources, Drawing Figs. 1, 11, and 5a. A.A.: Formal analysis, Investigation, Resources. A.S.: Supervision. S.H.T.: Project administration, Advisor, Resources, Editing.

\section{Competing interests}

The authors declare no competing interests.

\section{Additional information}

Supplementary Information The online version contains supplementary material available at https:/doi.org/ 10.1038/s41598-021-95683-3.

Correspondence and requests for materials should be addressed to R.P. or S.H.T.

Reprints and permissions information is available at www.nature.com/reprints.

Publisher's note Springer Nature remains neutral with regard to jurisdictional claims in published maps and institutional affiliations.

(c) (i) Open Access This article is licensed under a Creative Commons Attribution 4.0 International License, which permits use, sharing, adaptation, distribution and reproduction in any medium or format, as long as you give appropriate credit to the original author(s) and the source, provide a link to the Creative Commons licence, and indicate if changes were made. The images or other third party material in this article are included in the article's Creative Commons licence, unless indicated otherwise in a credit line to the material. If material is not included in the article's Creative Commons licence and your intended use is not permitted by statutory regulation or exceeds the permitted use, you will need to obtain permission directly from the copyright holder. To view a copy of this licence, visit http://creativecommons.org/licenses/by/4.0/.

(C) The Author(s) 2021 